\title{
Low dose inocula of SARS-CoV-2 B.1.1.7 variant initiate more robust infections in the upper respiratory tract of hamsters than earlier D614G variants
}

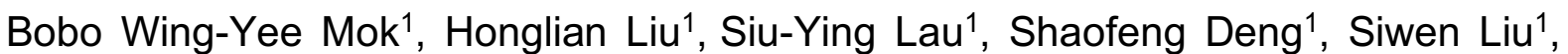
Rachel Chun-Yee Tam¹, Timothy Ting-Leung $\mathrm{Ng}^{2}$, Jake Siu-Lun Leung ${ }^{2}$, Pui Wang ${ }^{1}$, Kelvin Kai-Wang To', Jasper Fuk-Woo Chan ${ }^{1}$, Kwok-Hung Chan ${ }^{1}$, Kwok-Yung Yuen ${ }^{1}$, Gilman Kit-Hang Siu${ }^{2}$ and Honglin Chen ${ }^{1 *}$

${ }^{1}$ Department of Microbiology and State Key Laboratory for Emerging Infectious Diseases, Li Ka Shing Faculty of Medicine, The University of Hong Kong, Hong Kong SAR, China.

${ }^{2}$ Department of Health Technology and Informatics, Faculty of Health and Social Sciences, The Hong Kong Polytechnic University, Hong Kong SAR, China

*Correspondence: hlchen@hku.hk

KEYWORDS: SARS-CoV-2, VOC, B.1.1.7, hamster infection model, replication fitness

\section{Abstract}

There is a lack of experimental evidence to explain how the B.1.1.7 variant spreads more quickly than pre-existing variants in humans. We found that B.1.1.7 displays increased competitive fitness over earlier D614G lineages in an in-vitro system. Furthermore, , we demonstrated that B.1.1.7 variant is able to replicate and shed more efficiently in the nasal cavity than other variants with lower dose and shorter duration of exposure. 
In late 2020, a novel SARS-CoV-2 variant of concern (VOC), VOC 202012/01 (lineage B.1.1.7) was identified in the United Kingdom. This B.1.1.7 variant containing multiple mutations in spike ${ }^{1}$ has become dominant in the UK and is now rapidly spreading across multiple countries ${ }^{2}$. It is thought that this VOC has the potential to spread more quickly and with higher mortality than the pandemic to date ${ }^{3}$. Recently, using multiple behavioural and epidemiological data sources, Davies et al. estimated that the VOC 202012/01 variant (lineage B.1.1.7) has a 43-90\% higher reproduction number than pre-existing variants in England ${ }^{4}$. In another study, Davies et al. indicated that among specimens collected in the UK in early 2021, higher concentrations of virus were found on nasopharyngeal swabs from B.1.1.7 infected individuals, as measured by Ct values from PCR testing ${ }^{5}$. However, there is a lack of experimental evidence to support the expectation that B.1.1.7 does indeed spread more quickly than pre-existing variants.

The B.1.1.7 variant of SARS-CoV-2 harbours 21 non-synonymous point mutations and 3 deletions in comparison to the reference genome (accession number: NC_0.45512.2). Of these, 8 mutations and 2 deletions are involved in changes in the spike protein, which interacts with the host cell receptor, angiotensin-converting enzyme 2 (ACE2), and mediates virus entry into host cells ${ }^{6}$. These spike mutations include the deletion $\Delta \mathrm{H} 69 / \Delta \mathrm{V} 70$, which has arisen in multiple independent lineages and is suggested to associate with increased infectivity and evasion of the immune response $^{7}$; the mutation $\mathrm{N} 501 \mathrm{Y}$, which enhances binding affinity for the human ACE2 receptor and therefore influences viral transmissibility ${ }^{8}$ 9; and the mutation $\mathrm{P} 681 \mathrm{H}$, which is adjacent to the S1/S2 furin cleavage site in spike and might have an impact on viral infectivity ${ }^{10,11}$.

A recent study indicated that the SARS-CoV-2 VOC carrying the 501Y mutation showed no higher infectivity in cell than ancestral D614G variants ${ }^{12}$. Likewise, we did not observe replication of the B.1.1.7 variant to be significantly enhanced over that of other tested variants at any of the selected time-points in Vero-E6 and Calu-3 cells (Extended Data Fig. 1), however, we did observe that B.1.1.7 dominates in competitive fitness assays. These comparisons of replication fitness between B.1.1.7 and earlier circulating strains were performed in Calu-3 cells through simultaneous co-infection at 
a 1:1 ratio with B.1.1.7 (accession number: MW856794) and another variant of the D614G lineage, either B.1-G (HK-95, accession number: MT835143) or B.1.GH (405, accession number: MW856793) (Fig. 1). After three rounds of consecutive passage at 72-hour intervals, the B.1.1.7 variant became dominant in both co-culture conditions, suggesting that the additional substitutions in replication fitness in cells.

Next, we set up a Syrian hamster infection study to evaluate if B.1.1.7 exhibits higher infectivity in vivo. 6-8-week-old male Syrian hamsters were intranasally infected with 50 microliters of different variants $\left(2 \times 10^{4} \mathrm{PFU} / \mathrm{ml}\right)$, which is equivalent to $1000 \mathrm{PFU}$ per inoculum, as indicated in Fig. 2A. Infectious viral titres in upper (nasal) and lower (pulmonary) tissues were measured on four consecutive days after infection. All viruses tested replicated to similar titres in nasal turbinate and lung tissues of infected hamsters. This result is consistent with two recent studies which also found no significant alteration in infectious viral titres in samples collected from nasal washes, throat swabs and lungs from hamsters infected with different SARS-CoV-2 variants ${ }^{13}$, 14. Given that hamsters are highly susceptible to SARS-CoV-2 infection, intranasal infection with high-titre inocula may hamper discrimination of differences in the infectivity and replication efficiency of variants ${ }^{15}$. In fact, by titrating the infection dosage (10-fold dilution) of the inocula administered to hamsters, we observed that viral replication in nasal tissues of infected hamsters had already plateaued with infection doses of 100 PFU and upwards, even on day one post-infection (Extended data Fig. 2). Humans are exposed to varying doses of infectious particles during SARS-CoV-2 transmission. We reasoned that SARS-CoV-2 variants which can initiate effective infection with fewer infectious particles are likely to transmit more effectively than other variants requiring more infectious particles. To test this, we performed another hamster infection study using only 10 PFU per inoculum, with samples being collected at 16 hours post-infection. Interestingly, infectious viral loads in nasal turbinates of hamsters were found to be significantly higher with B.1.1.7 compared to the other viruses, whereas similar viral loads were observed in lungs of all infected hamsters, except for those inoculated with B.1-G (HK-95), which exhibits higher viral titres in lungs, although with large variations between replicates (Fig. 2B). 
102 SARS-CoV-2 VOCs have been emerging in different countries in the past few months,

103 and it is crucial to establish relevant experimental models to characterise existing and

104 new variants in terms of transmissibility, disease severity and vaccine efficacy, and to 105 evaluate therapeutic interventions. In this report, by using a lower infectious dose, we 106 demonstrate that B.1.1.7 exhibits higher infectivity and/or replication efficiency in the 107 nasal epithelium. Our data, albeit limited, strengthen the contention that this novel 108 VOC is more easily transmitted than other pre-existing strains. Further work, including 109 transmission studies with optimised inoculum dosages and timing of sample collection 110 and investigation into routes of transmission are required. A better understanding of 111 SARS-CoV-2 dynamics is important for designing combative strategies for the 112 prevention and control of virus infections. 
118 1. England, P.H. Investigation of novel SARS-CoV-2 Variants of Concern. https://www.gov.uk/government/publications/investigation-of-novel-sars-cov-2variant-variant-of-concern-20201201 (2020).

2. O'Toole, A., Hill, V., Pybus, O.G. Tracking the international spread of SARSCoV-2 lineages B.1.1.7 and B.1.351/501Y-V2. https://virological.org/t/trackingthe-international-spread-of-sars-cov-2-lineages-b-1-1-7-and-b-1-351-501y$\underline{v 2 / 592}(2021)$.

3. Grint, D.J. et al. Case fatality risk of the SARS-CoV-2 variant of concern B.1.1.7 in England. 2021.2003.2004.21252528 (2021).

4. Davies, N.G. et al. Estimated transmissibility and impact of SARS-CoV-2 lineage B.1.1.7 in England. Science (2021).

5. Davies, N.G. et al. Increased mortality in community-tested cases of SARSCoV-2 lineage B.1.1.7. Nature (2021).

6. Starr, T.N. et al. Deep mutational scanning of SARS-CoV-2 receptor binding domain reveals constraints on folding and ACE2 binding. bioRxiv (2020).

7. Kemp, S. et al. Recurrent emergence and transmission of a SARS-CoV-2 Spike deletion H69/V70. 2020.2012.2014.422555 (2021).

8. Dejnirattisai, W. et al. The antigenic anatomy of SARS-CoV-2 receptor binding domain. Cell (2021).

9. Tian, F. et al. Mutation N501Y in RBD of Spike Protein Strengthens the Interaction between COVID-19 and its Receptor ACE2. 2021.2002.2014.431117 (2021).

10. Hoffmann, M., Kleine-Weber, H. \& Pohlmann, S. A Multibasic Cleavage Site in the Spike Protein of SARS-CoV-2 Is Essential for Infection of Human Lung Cells. Mol Cell 78, 779-784 e775 (2020).

11. Seyran, M. et al. The structural basis of accelerated host cell entry by SARSCoV-2†. Febs j (2020).

12. Li, Q. et al. SARS-CoV-2 501Y.V2 variants lack higher infectivity but do have immune escape. Cell (2021).

13. Abdelnabi, R. et al. Comparative infectivity and pathogenesis of emerging SARS-CoV-2 variants in Syrian hamsters. 2021.2002.2026.433062 (2021).

14. Mohandas, S. et al. Comparison of the pathogenicity and virus shedding of SARS CoV-2 VOC 202012/01 and D614G variant in hamster model. 2021.2002.2025.432136 (2021). 
15. Imai, M. et al. Syrian hamsters as a small animal model for SARS-CoV-2 infection and countermeasure development. Proc Natl Acad Sci U S A 117, 16587-16595 (2020).

Acknowledgments

175

176 The authors would like to thank Dr Jane Rayner for critical reading and editing of the

177 manuscript. This study is partly supported by the Theme-Based Research Scheme

178 (T11/707/15) and General Research Fund (17107019) of the Research Grants Council,

179 Hong Kong Special Administrative Region, China, and the Sanming Project of

180 Medicine in Shenzhen, China (No. 290 SZSM201911014).

181

182

\section{Declaration of interest statement}

184

185 No potential conflict of interest was reported by the author(s). 
187 Figure 1. In-vitro Competitive Fitness Assay. Sanger sequencing chromatograms of spike gene fragments amplified from viral samples in the competition assay. Cell cultures were infected with a 1:1 mixture of two variants, as indicated, at an $\mathrm{MOI}$ of 0.1. The supernatants were serially passaged three times in Calu-3 cells. $901 \mathrm{bp}$ fragments containing residue 501 (boxed) were amplified from the vRNA of individual samples collected from each passage $(P)$ and sequenced. B.1-G (HK-95) and B.1.GH (405) are $501 \mathrm{~N}, \mathrm{~B} \cdot 1.1 .7$ is $501 \mathrm{Y}$.

Figure 2. In-vivo Infection Studies. Viral replication of different SARS-CoV-2 variants in nasal turbinates and lungs of hamsters. Hamsters were infected with different SARS-CoV-2 variants, as indicated. Viral titers in nasal turbinates and lungs were determined by plaque assay (PFU/ml). (A) Hamsters (14 per variant virus group) were each inoculated intranasally with 50 ul of virus stock containing 1000 PFU of virus. Three to four hamsters from each group were euthanized on each of the four consecutive days following infection for viral titration. (B) Hamsters (4-5 per group) were each inoculated intranasally with 50 ul of virus stock containing 10 PFU of virus. One non-D614G lineage variant (HK-15 (MT835141)) and three D614G lineage variants (GH (405), B.1.1.7 and G (HK-95)) were used. Hamsters were euthanized at 16 hours post-infection for viral titration. Horizontal lines indicate the overall mean of average viral titer values per group. Statistical significance was calculated by Student's t-test; * denotes $p<0.05,{ }^{* * *}$ denotes $p<0.0005$ and ns denotes nonsignificant. 


\section{Figure 1}

217

G (HK-95) vs B.1.1.7 (1:1)

A A T TA T

$(\mathrm{N}) \rightarrow(\mathrm{Y})$

PO

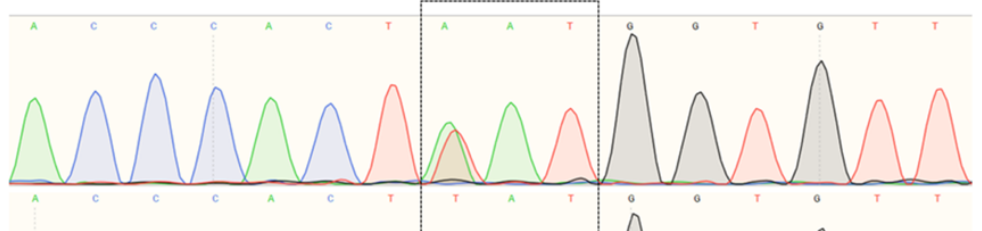

P1

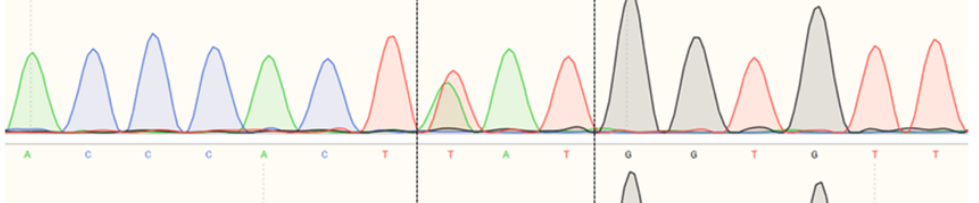

P2

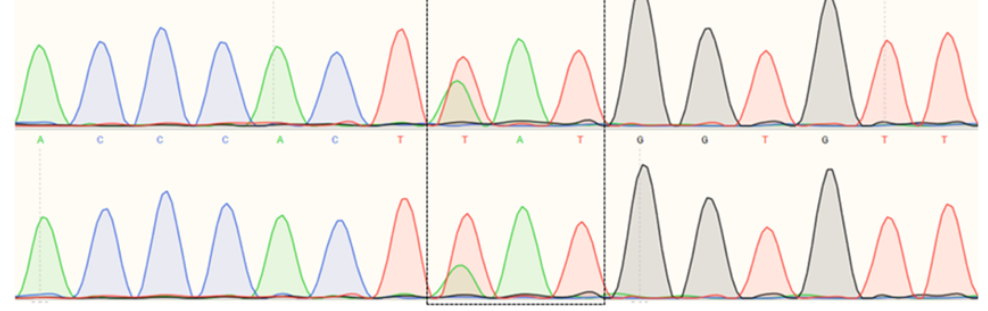

GH (405) vs B.1.1.7 (1:1)

A A T TAT

$(\mathrm{N}) \rightarrow(\mathrm{Y})$

PO

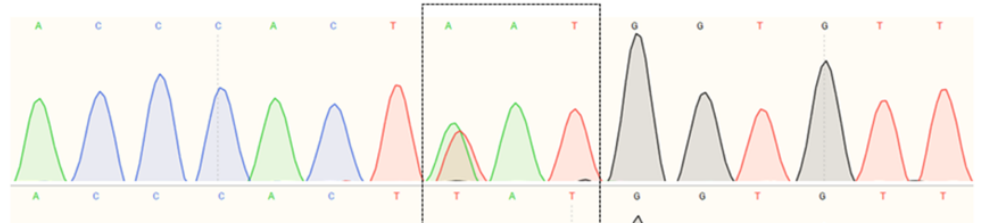

1

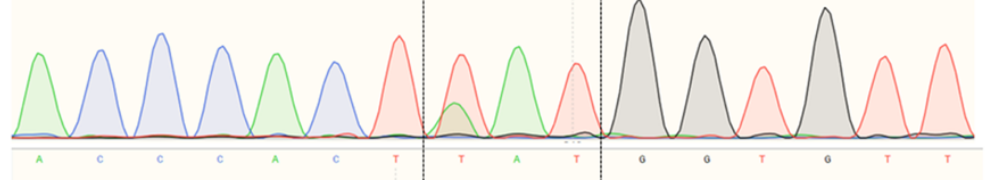

P3

P2
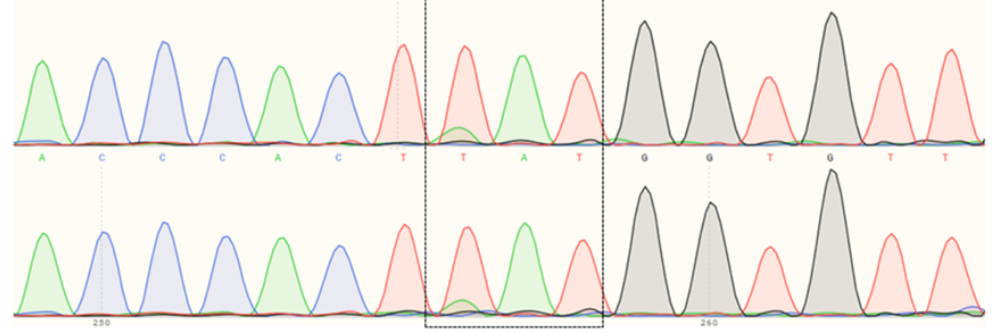
A Nasal Turbinate (1000 PFU)

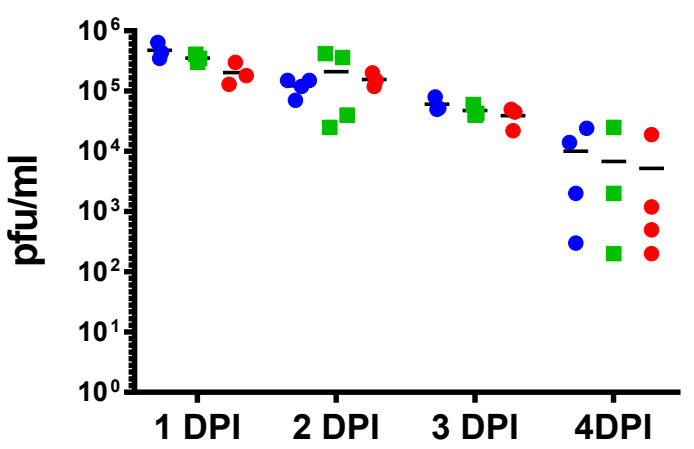

B Nasal Turbinate (10 PFU / 16 hpi)

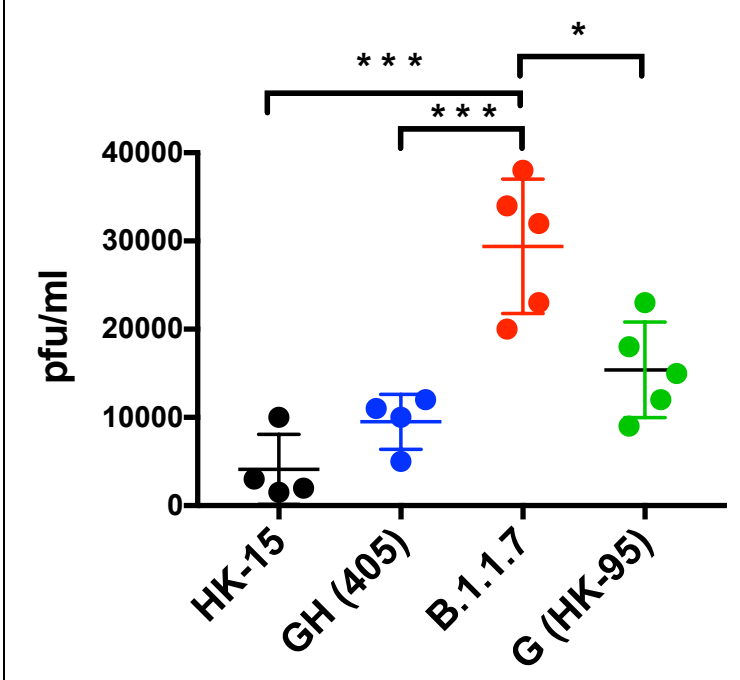

Lung (1000 PFU)

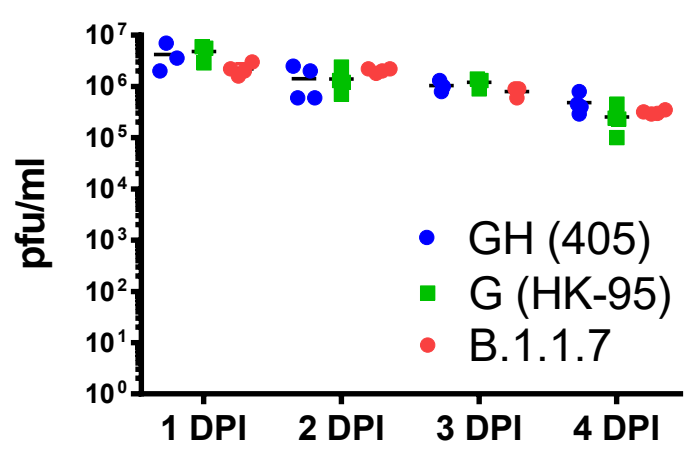

Lung (10 PFU / 16 hpi)

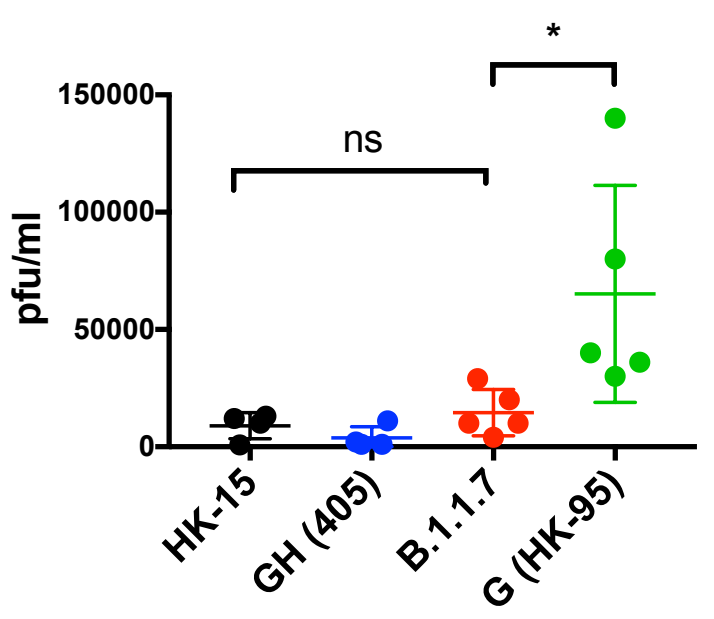




\section{Methods}

\section{Viruses}

The SARS-CoV-2 isolates HK-95 (MT835143), 405 (MW856793), B.1.1.7 (MW856794) and HK-15 (MT835141) were isolated from specimens obtained from four laboratoryconfirmed COVID-19 patients using Vero E6 cells (ATCC; CRL-15786). All experiments involving SARS-CoV-2 viruses were conducted in a Biosafety Level-3 laboratory. For animal challenge, viral stocks were prepared after two serial passages of isolated virus in Vero E6 cells in Dulbecco's Modified Eagle Medium (DMEM) (Thermo Fisher Scientific) supplemented with 5\% fetal bovine serum (Thermo Fisher Scientific), and $100 \mathrm{IU}$ penicillin $\mathrm{G} / \mathrm{ml}$ and $100 \mathrm{ml}$ streptomycin sulfate/ml (Thermo Fisher Scientific). Virus titres were then determined by plaque assay using Vero E6 cells. Viral RNAs were obtained from the supernatants of infected cells and then isolated using the QIAamp RNA Viral kit (Qiagen) and subjected to whole viral genome sequencing.

\section{Hamster Infection}

244 Female golden Syrian hamsters, aged 6-8 weeks old, were obtained from the LASEC,

245 Chinese University of Hong Kong via the Centre for Comparative Medicine Research 246 at the University of Hong Kong (HKU). All experiments were performed in a Biosafety

247 Level-3 animal facility at the LKS Faculty of Medicine, HKU. All animal studies were 248 approved by the Committee on the Use of Live Animals in Teaching and Research, 249 HKU. Hamsters were anesthetized with ketamine $(150 \mathrm{mg} / \mathrm{kg})$ and xylazine $(10 \mathrm{mg} / \mathrm{mg})$ 250 via intraperitoneal injection prior to nasal inoculation. All hamsters were euthanized by intraperitoneal injection of pentobarbital at $200 \mathrm{mg} / \mathrm{kg}$. 


\section{In-vitro Competitive Fitness Assay}

258 Calu-3 cells in Dulbecco's Modified Eagle Medium (DMEM) (Thermo Fisher Scientific)

259 supplemented with 5\% fetal bovine serum (Thermo Fisher Scientific), and 100 IU 260 penicillin $\mathrm{G} / \mathrm{ml}$ and $100 \mathrm{ml}$ streptomycin sulfate/ml (Thermo Fisher Scientific) were

261 infected with $\mathrm{MOI}$ of 0.1 of B.1.1.7 and another variant of the D614G lineage, either 262 B.1-G (HK-95) or B.1.GH (405) mixture at 1:1 ratios. Following $1 \mathrm{~h}$ incubation, the 263 cultures were washed thrice with PBS and cultures for 3 days. To passage the progeny 264 viruses, the virus samples were continuously passaged three times in Calu-3 cells. 265 Viral RNAs were obtained from the supernatants of infected cells and then isolated 266 using the QIAamp RNA Viral kit (Qiagen). A $901 \mathrm{bp}$ fragment containing the N501Y 267 site was amplified from each RNA sample by RT-PCR using primer set: 5'268 GAAGTCAGACAAATCGCTCCAG-3' and 5'-GCAACTGAATTTTCTGCACCA-3'. The amplicon was purified

by

270 NucleoSpin $®$ Gel and PCR Clean-Up (Takara) for Sanger sequencing. 